Original Article

\title{
Diabetic Macular Edema Patients with Poor Baseline Visual Acuity Treated with Ranibizumab in Real Life and Optical Coherence Tomography Based Predictor Factors for Visual Outcomes
}

\author{
Abdullah Ozkaya, MD1, Mehmet Ozveren, MD¹, Okkes Baz, MD¹, Hatice Nur Tarakcioglu, MD¹, \\ Korhan Fazil, MD ${ }^{1}$, M uhittin Taskapili, MD $^{1}$ \\ ${ }^{1}$ Beyoglu Eye Training and Research Hospital, Bereketzade Cami Sok., 34421 Beyoglu, Istanbul, Turkey. \\ Corresponding Author: Abdullah Ozkaya, Beyoglu, Eye Training and Research Hospital, Bereketzade Cami Sok., 34421 Beyoglu, Istanbul, \\ Turkey.Phone:00902122515900 E-mail : abdozkaya@gmail.com
}

$\begin{array}{ll}\text { Received } & : 13.10 .2017 \\ \text { Review Completed } & : 02.02 .2018 \\ \text { Accepted } & : 10.05 .2018\end{array}$

Keywords : Diabetic macular edema; intravitreal injection; ranibizumab, visual acuity.

\begin{tabular}{|c|}
\hline Access this article online \\
\hline Quick Response Code \\
\hline
\end{tabular}

\begin{abstract}
:
Purpose: We aimed to evaluate the real life outcomes of ranibizumab in the treatment of diabetic macular edema (DM E) patients with a baseline visual acuity $\varangle 0.05$ in decimals.

Methods: Newly diagnosed DM E patients with a visual acuity $\leq 0.05$, treated with ranibizumab monotherapy, and completed a follow-up time of 12 months were included retrospectively. Patients were evaluated in regards to change in best corrected visual acuity (BCVA) and central retinal thickness, and the total visit and injection numbers.
\end{abstract}

Results: A total of 24 eyes of 24 patients were included. Mean BCVA at baseline, month 3, 6, 9, and 12 was, $0.04 \pm 0.01,0.12 \pm 0.12,0.12 \pm 0.11,0.17 \pm 0.19$, and $0.21 \pm 0.21$ ( $p \varangle 0.05$ for all), respectively. One eye (4.2\%) had VA loss of $\geq 3$ lines, and six eyes ( $25.0 \%$ ) had stable vision (loss of $<3$ line, or remained stable, or gained $<1$ lines), and 17 eyes (70.8\%) had VA gain of $\geq 3$ linesat month 12.The mean visit number at month 12 was $4.8 \pm 1$ and the mean injection number was $4.0 \pm 1.4$.

Conclusion: Ranibizumab seemed to be effective in the treatment of DM E patients with a low visual acuity in real life.

\section{Introduction}

Diabetic retinopathy (DR) is the most frequent retinal vascular disease and diabetic macular edema (DME) is an important cause of visual loss among the DR patients (1-5). Laser photocoagulation, vitrectomy, and intravitreal injections of different drugs were used in the treatment of DME (2-5). Intravitreal injection of anti-vascular endothelial growth (Anti-VEGF) agents is the most preferred treatment modality currently (4-9). Ranibizumab was proven to be effective in the treatment of DME with various treatment regimens which were monthly, pro re nata (PRN), treat and extend etc (4-9). In prospective multicenter studies it was reported that, a mean of 8-9 ranibizumab injections were required in the first 12 months of treatment; however, the mean injection number dramatically decreased after the first year and nearly no injections were needed at year 4 and 5 (4-7). It was not possible to follow the strict follow-up and retreatment criteria of these studies in real life especially when PRN regimen was preferred $(8,9)$. Also, only the patients with a VA between 20/320 and 20/32-20/40 were included in these efficacy studies, and the patients with better or worse levels were not evaluated usually (4-8). In addition, it is a known fact that some optical tomography (OCT) markers such as the presence of an intact inner segment/outer segment (IS/OS) junction, disorganization of the retinal inner layers (DRILL), and the presence of subretinal fluid was found to be associated with the visual outcomes of DME treatment (10-17). Therefore in this study, we aimed to evaluate the efficacy of intravitreal ranibizumab (IVR) on a PRN treatment regimen in DME patients with a VA $\leq 0.05$ in decimals and OCT based predictor factors for the treatment outcome. 


\section{Materials and Methods}

In this retrospective study, medical records of the patients who had DME and underwent IVR treatment on a PRN treatment regimen between January 2013 and December 2015 were analyzed. Newly diagnosed treatment naïve DME patients with non-proliferative DR and a best corrected visual acuity $\leq 0.05$ in decimals, and who completed a follow time of 12 months in our clinic were included. The patients with a history of any other treatment for DM E at the first admission, or who were lost to follow-up, or received any other treatment for DME during the first 12 months of our follow-up were not included. A patient database of 1182 DME patients was reviewed for this study. A hundred and sixty-eight patients were treated with ranibizumab monotherapy and completed the minimum follow-up period of 12 months. Twenty-four eyes of 24 patients out of these 168 patients met the inclusion criteria and were included for this study. A written informed consent was obtained from all patients before the treatment. The study adhered to the tenets of the Declaration of Helsinki.

Data collected from the patients' records included age, gender, best corrected visual acuity (BCVA), central retinal thickness (CRT) at the baseline, and at month 3, 6, 9, and 12. Visit and injection numbers during the first 12 months were also recorded.

All patients underwent a standardized examination including measurement of BCVA via a projection chart in decimals at 4 meters, slit-lamp bio-microscopy, measurement of IOP via applanation tonometry, and biomicroscopic fundus examination. Fundus photography, fluorescein angiography (FA) (HRA-2; Heidelberg Engineering, Heidelberg, Germany), and OCT imaging (Spectralis; Heidelberg Engineering, Heidelberg, Germany) were performed before treatment. All examinations were repeated monthly, except for FA. Fluorescein angiography was repeated according to the physicians' discretion. Optical coherence tomography was used for detecting macular edema and measurement of CRT. Central retinal thickness, defined as the mean thickness of the neuro sensory retina in a central $1 \mathrm{~mm}$ diameter area, was computed using OCT mapping software generated by the device. Diabetic macular edema was diagnosed via FA and OCT, and patients with a CRT of $>300$ microns were considered to have DME. The severity of non-proliferative DR, angiographic classification of DME was not assessed. The presence of macular ischemia was evaluated in the included eyes. M acular ischemia was diagnosed when the longest diameter of foveal avascular zone (FAZ) was $>1000$ $\mu$ measured via the built in caliper software of the device. On the contrary, FAZ was accepted normal FAZ if the longest diameter was $<1000 \mu$ with a regular and round/horizontally oval shape (18). The patients were divided into two groups in regard to the increase in BCVA at month 12. The first group consisted of the patients who had a gain in BCVA $\geq 3$ LogM AR line, and the second group consisted of the patients who had a gain $<3$ LogM AR line or lost vision. These two groups were compared in regards to OCT parameters which were the integrity of the IS/OS junction (10), the presence of cystic macular edema (11), DRILL (12), and subfoveal sensorial retinal detachment (10, 11,13 ). Integrity of the IS/OS junction was classified into two subgroups semi quantitatively by eye examination; if the IS/OS junction was intact and clearly visible under the OCT section which trans-passed through the foveal depression then the IS/OS junction was called intact, if there was a definite continuity defect in the junction then it was called disrupted. The classification of DME subtype according to the intra retinal cyst patterns were divided into two groups; if the diameter of the largest intra retinal cyst was $<300$ micron then the DME was classified as spongiform edema, if the diameter of the largest intra retinal cyst was $>300$ microns then the DM E was classified as cystic edema (11). Presence of DRILL, and subfoveal sensorial retinal detachment were all classified into two groups as present of absent (10-13). The patients were divided into two groups according to the increase in BCVA and the percentage of presence of the OCT parameters were compared between these two groups. The patients who gained $\geq 3$ lines of vision were classified as group 1 , and the patients who showed $<3$ lines gain in vision or lost 
vision were classified as group 2.

All injections were performed under sterile conditions after application of topical anesthesia, use of $10 \%$ povidone-iodine (Betadine; Purdue Pharma, Stamford, CT, USA) scrub was used on the lids and lashes, and 5\% povidone-iodine was administered on the conjunctival sac. Intravitreal ranibizumab $0.5 \mathrm{mg} / 0.05 \mathrm{ml}$ (Lucentis; Novartis, Basel, Switzerland) was injected through the pars plana at $3.5 \mathrm{~mm}$ posterior to the limbus with a 30 -gauge needle. Patients were instructed to admit back the hospital if they experienced decreased vision, eye pain, or any new arising symptoms.

Initially, all of the patients were prescribed to receive a loading dose of three consecutive monthly injections. Then the patients were followed monthly, and a single injection of IVR was repeated when the VA decreased by one or more lines, or an increase of $>100$ microns in CRT in OCT images compared to the last visit.

Primary outcome measures of this study included the change in BCVA and CRT. Secondary outcome measure was the predictive factors in OCT for the visual improvement.

\section{Statistical Analysis}

Visual acuity was converted to the logarithm of the minimum angle of resolution (LogMAR) for statistical analysis. Categorical variables were presented as numbers and percentages, while numerical variables were expressed as the mean and standard deviation. First the data was analyzed in terms of normality using Shapiro-Wilk test. As the distribution of the data was found to be normal, the visual acuity and the CRT values between baseline and the other time points were assessed with repeated measures test. The means within the groups were compared using independent sample t-test. Categorical variables were compared using chi-square test. A p value $\varangle$.05 was considered statistically significant.

\section{Results}

A total of 24 eyes of 24 patients were included. The mean age was $59.2 \pm 9.3$ years (range $36-73$ years) and 15 patients (62.5\%) were female; nine patients (37.5\%) were male.
General characteristics of the patients were summarized in table 1.

The mean BCVA at baseline was $0.04 \pm 0.01$ in decimals (range 0.01-0.05). The BCVA at month 3, 6, 9, 12 was $0.12 \pm 0.12$ (range 0.01-0.5) $(p=0.009), 0.12 \pm 0.11$ (range $0.02-0.5) \quad(p=0.002), 0.17 \pm 0.19$ (range $0.02-0.8$ ) $(p<0.0001$ ), and $0.21 \pm 0.21$ (range 0.02-1.0) $(p<0.0001$ ), respectively. One eye (4.2\%) had VA loss of $\geq 3$ lines, and six eyes $(25.0 \%)$ had stable vision (loss of $<3$ line, or remained stable, or gained $<1$ lines), and 17 eyes $(70.8 \%)$ had VA gain of $\geq 3$ lines at month 12 . In regard to visual outcomes at month 12,17 patients $(70.8 \%)$ were included in group 1 and 7 patients (29.2\%) were included in group 2 . The IS/OS junction was intact in 13 of 17 eyes (76.5\%) in group 1, and 3 of 7 eyes ( $42.9 \%$ ) in group $2(p=0.1)$. Cystic macular edema was detected in 15 of 17 eyes (88.2\%) in group 1 and 6 of 7 eyes (85.7\%) in group $2(p=0.9)$. DRILL was detected in 11 of 17 eyes (64.7\%) in group 1 and 4 of 7 eyes $(57.1 \%)$ in group $2(p=0.9)$. Subfoveal sensorial retinal detachment was present in 9 of 17 eyes (52.9\%) in group 1 and in 1 of 7 eyes (14.4\%) in group $2(p=0.1)$. Macular ischemia was present in 4 of 17 eyes (23.5\%) in group 1 and in 5 of 7 eyes ( $71.4 \%$ ) in group $2(p=0.02)$.

M ean CRT at baseline was $465 \pm 150$ microns (range 320759). The CRT at month $3,6,9,12$ was $364 \pm 119$ (range 216$677)(p=0.1), 394 \pm 131$ (range 233-754) $(p<0.053), 383 \pm 121$ (range 225-652) $(p=0.1)$, and 330 \pm 102 (range 201-586) $(p=0.004)$, respectively. At month 12,14 of the 15 eyes (62.5\%) had a CRT $<350$ microns.

The mean planned visit number at month 12 was $5.1 \pm 0.8$ (range 4-7), and the number of completed visits were $4.8 \pm 1.0$ (range 3-7) (94.3\% completion). The mean number of planned injections at month 12 was $4.2 \pm 1.7$ (range 1-8), and the number of performed injections were $4.0 \pm 1.4$ (range 1-7) (92.3\% completion).

No injection related endophthalmitis was noted after a total of 96 injections. 
Table 1 : General characteristics of the patients

\begin{tabular}{|l|c|}
\hline Number of eyes & 24 \\
\hline Age (years) & $59.2 \pm 9.3$ \\
\hline Gender (male/female) & $9 / 15$ \\
\hline Lens status (phakic/pseudophakic) & $18 / 6$ \\
\hline Baseline BCVA (in decimals) & $0.04 \pm 0.01$ \\
\hline Baseline CRT (microns) & $465 \pm 150$ \\
\hline
\end{tabular}

BCVA : best corrected visual acuity; CRT: central retinal thickness.
Table 2 : The mean best corrected visual acuity and central retinal thickness levels at different time points.

\begin{tabular}{|l|c|c|c|c|c|}
\hline & Baseline & Month 3 & Month 6 & Month 9 & M onth 12 \\
\hline $\begin{array}{l}\text { BCVA, in decimals } \\
\text { (LogM AR) }\end{array}$ & $0.04 \pm 0.01$ & $0.12 \pm 0.12$ & $0.12 \pm 0.11$ & $0.17 \pm 0.19$ & $0.21 \pm 0.21$ \\
$(1.41 \pm 0.24)$ & $(1.05 \pm 0.37)$ & $(1.03 \pm 0.36)$ & $(0.95 \pm 0.42)$ & $(0.84 \pm 0.41)$ \\
\hline CRT, microns & $465 \pm 150$ & $364 \pm 119$ & $394 \pm 131$ & $383 \pm 121$ & $330 \pm 102$ \\
\hline $\begin{array}{l}\text { Number of } \\
\text { injections }\end{array}$ & - & - & - & - & $4.0 \pm 1.4$ \\
\hline
\end{tabular}

BCVA, best corrected visual acuity; CRT, central retinal thickness

Table 3 : General data of the whole study population.

\begin{tabular}{|c|c|c|c|c|c|c|c|c|c|c|c|c|c|c|c|c|c|c|c|c|}
\hline Patien & Gender & Age, & aselir & Mont & Month & Month & Month & Baseline & Month & Month & Month & Month & Visit & Inj. & Severity & Macula & IS/OS & $\mathrm{CME}$ & RILL & SRD \\
\hline no. & & years & VA & $3 \mathrm{VA}$ & $6 \mathrm{VA}$ & 9 VA & $12 \mathrm{VA}$ & CRT, $\mu$ & 3 CRT, $\mu$ & 6 CRT, & 9 CRT, $\mu$ & 12 CRT, & no. & no. & of NPDR & Ishemia & & & & \\
\hline 1 & Female & 66 & 0.02 & 0.02 & 0.02 & 0.05 & 586 & 439 & 469 & 754 & 603 & 586 & 5 & 6 & Severe & + & Disrupted & + & + & - \\
\hline 2 & Female & 59 & 0.03 & 0.25 & 0.10 & 0.10 & 317 & 732 & 255 & 362 & 422 & 317 & 4 & 3 & Severe & - & Normal & + & + & + \\
\hline 3 & Male & 56 & 0.05 & 0.05 & 0.16 & 0.50 & 252 & 320 & 294 & 288 & 276 & 252 & 5 & & Moderate & - & Normal & - & - & + \\
\hline 4 & Male & 55 & 0.01 & 0.16 & 0.20 & 0.10 & 344 & 346 & 333 & 449 & 444 & 344 & 5 & 2 & Severe & + & Disrupted & + & + & - \\
\hline 5 & Female & 65 & 0.01 & 0.01 & 0.05 & 0.05 & 423 & 655 & 398 & 547 & 396 & 423 & 7 & 5 & Severe & - & Normal & + & - & + \\
\hline 6 & Female & 63 & 0.01 & 0.40 & 0.20 & 0.40 & 231 & 499 & 322 & 294 & 283 & 231 & 3 & 5 & Severe & - & Normal & + & + & + \\
\hline 7 & Male & 55 & 0.05 & 0.05 & 0.50 & 0.80 & 268 & 704 & 335 & 604 & 269 & 268 & 4 & & Moderate & - & Normal & - & - & - \\
\hline 8 & Female & 59 & 0.05 & 0.50 & 0.05 & 0.10 & 486 & 612 & 216 & 388 & 652 & 486 & 6 & 4 & Severe & + & Disrupted & + & + & - \\
\hline 9 & Female & 73 & 0.05 & 0.05 & 0.05 & 0.05 & 320 & 324 & 343 & 366 & 384 & 320 & 4 & 3 & Severe & + & Disrupted & + & + & - \\
\hline 10 & Female & 63 & 0.05 & 0.05 & 0.05 & 0.10 & 236 & 380 & 221 & 276 & 355 & 236 & 5 & 2 & Severe & + & Normal & + & + & - \\
\hline 11 & Male & 68 & 0.05 & 0.05 & 0.10 & 0.05 & 310 & 370 & 372 & 350 & 313 & 310 & 5 & 3 & Severe & - & Normal & + & - & - \\
\hline 12 & Female & 67 & 0.05 & 0.10 & 0.05 & 0.16 & 295 & 382 & 312 & 344 & 323 & 295 & 4 & 4 & Moderate & - & Disrupted & + & - & - \\
\hline 13 & Female & 51 & 0.05 & 0.10 & 0.05 & 0.05 & 491 & 370 & 362 & 414 & 635 & 491 & 4 & 4 & Moderate & + & Disrupted & + & - & - \\
\hline 14 & Male & 59 & 0.05 & 0.10 & 0.05 & 0.10 & 471 & 323 & 677 & 504 & 575 & 471 & 6 & 7 & Severe & - & Normal & - & + & - \\
\hline 15 & Female & 73 & 0.05 & 0.05 & 0.05 & 0.05 & 407 & 330 & 396 & 284 & 316 & 407 & 4 & 3 & Severe & + & Normal & + & + & - \\
\hline 16 & Male & 59 & 0.05 & 0.10 & 0.05 & 0.05 & 327 & 326 & 299 & 279 & 367 & 327 & 5 & 4 & Severe & + & $\mid$ Disrupted & + & + & - \\
\hline 17 & Female & 56 & 0.05 & 0.05 & 0.05 & 0.02 & 201 & 368 & 227 & 270 & 225 & 201 & 5 & 2 & Moderate & + & Disrupted & + & - & - \\
\hline 18 & Female & 55 & 0.05 & 0.05 & 0.20 & 0.40 & 233 & 412 & 275 & 233 & 441 & 233 & 6 & 5 & Severe & - & Normal & + & - & - \\
\hline 19 & Male & 66 & 0.05 & 0.20 & 0.20 & 0.32 & 290 & 687 & 574 & 468 & 333 & 290 & 6 & & Moderate & - & Normal & + & + & + \\
\hline 20 & Female & 36 & 0.05 & 0.20 & 0.16 & 0.10 & 228 & 759 & 388 & 417 & 273 & 228 & 6 & 5 & Severe & - & Normal & + & + & + \\
\hline 21 & Female & 62 & 0.05 & 0.25 & 0.32 & 0.20 & 284 & 392 & 607 & 611 & 304 & 284 & 4 & & Moderate & - & Normal & + & + & + \\
\hline 22 & Male & 68 & 0.05 & 0.05 & 0.05 & 0.16 & 298 & 343 & 402 & 356 & 350 & 298 & 3 & 4 & Severe & - & Normal & + & + & + \\
\hline 23 & Female & 36 & 0.05 & 0.10 & 0.16 & 0.32 & 223 & 614 & 265 & 272 & 271 & 223 & 6 & 41 & Moderate & - & Normal & + & + & + \\
\hline 24 & Male & 53 & 0.05 & 0.10 & 0.05 & 0.05 & 409 & 476 & 399 & 326 & 404 & 409 & 4 & 41 & Moderate & - & Normal & + & - & + \\
\hline
\end{tabular}

Abbreviations: VA, visual acuity (in decimals); CRT, central retinal thickness; $\mu$, micrometer; no, number; inj, injection; NPDR, nonproliferative diabetic retino pathy; IS/OS, inner segment-outer segment junction; CM E, cystic macular edema; DRILL, disorganization of the retinal inner layers; SRD, sensorial retinal detachment

Figure 1 : Dot-blot diagram of visual acuity levels at different time points versus baseline, a) baseline visual acuity versus month 3 , b) baseline visual acuity versus month $6, c)$ baseline visual acuity versus month 12. (VA, visual acuity; visual acuity levels were expressed in decimals)

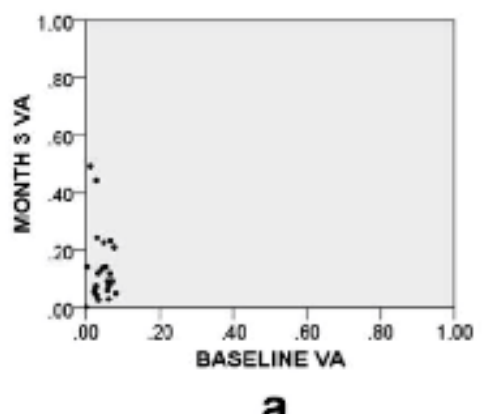

a
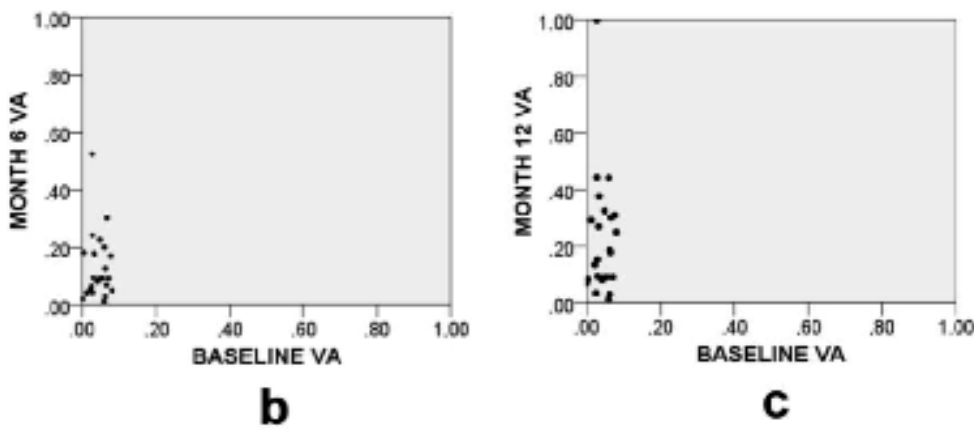
Figure 2: Dot-blot diagram of central retinal thickness levels at different time points versus baseline, a) baseline central retinal thickness versus month 3, b) baseline central retinal thickness versus month $6, \mathrm{c}$ ) baseline central retinal thickness versus month 12 . (CRT, central retinal thickness; central retinal thickness levels were expressed in micrometers)

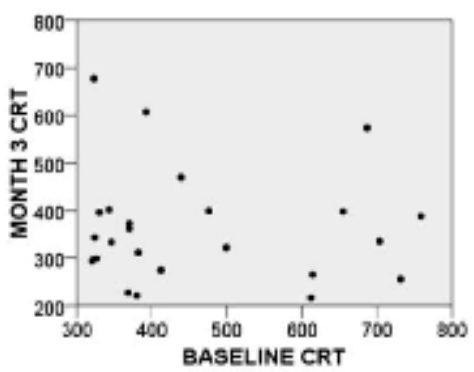

a

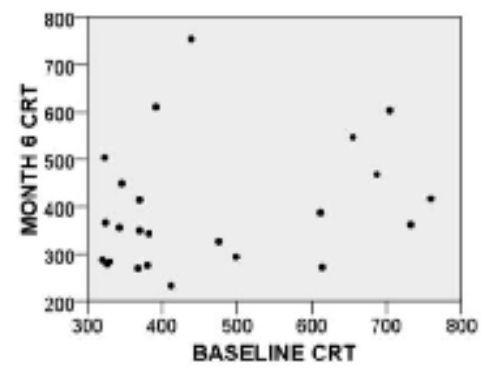

b

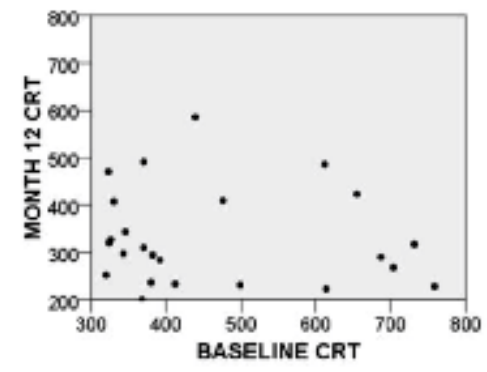

C

\section{Discussion}

There were a few studies in the literature regarding the outcomes of anti-VEGF treatment in neo vascular AMD patients with a poor visual acuity (14). However, a literature search from PubMED did not reveal such a focused study in DM E patients.

In this study we evaluated the treatment outcomes, visit and injection numbers, and OCT parameters of ranibizumab treated DME patients with a poor baseline visual acuity. As our study was a real life practice the visit and injection numbers were very low in contrast to previous prospective studies. In spite of this low injection number the visual achievements were fairly good as 17 of 24 eyes (70.8\%) gained $>3$ LogMAR lines of vision. The mean increase in BCVA was 1.7 Decimal lines in Decimals and 6.4 LogM AR which was also a satisfactory result. The decrease in mean CRT was statistically significant at least at the last visit at month 12. Several OCT markers were evaluated in the literature in regard to predict the visual outcomes of the DME patients (10-13,15-17). Seo et al, evaluated the visual and morphological outcomes of IVR treatment in patients with DME in regard to OCT based DM E patterns (13). They classified the included 55 eyes into three subgroups as patients with diffuse retinal thickening, cystoid macular edema, and serous retinal detachment. They reported that the mean required injection numbers during the 12 months of follow up was different among the two groups. The cystoid macular edema group required 5.33 , the serous detachment group required 5.03, and the diffuse thickening group required 3.69 injections. Also they mentioned that disruption of the photoreceptor integrity at baseline was found to be correlated with poorer visual outcome which was detected more frequently in serous detachment group. Sophie et al, investigated the predictors of functional and anatomical outcomes in DME patients treated with ranibizumab for 24 months of treatment (11). They evaluated several parameters in ranibizumab treated and sham treated patients and concluded that the presence of sub macular fluid, intra retinal cysts, severe macular thickening, and renal disease were the factors which were found to be associated with poor visual outcomes in sham treated patients and which responded well to ranibizumab treatment. The associations of DRILL with visual outcomes in DME patients were evaluated in a study by Radwan et al (12). They evaluated the visual outcomes of the macular edema patients who showed DRILL at the beginning of the treatment and graded these patients in regard to the change in DRILL length in central macular region after 8 months after the resolution of DM E. The included patients were divided into two groups as the patients who showed resolution of DRILL, and who did not. The authors of the study concluded that the resolution of DRILL after treatment was associated with increased vision. In another study regarding the association between hyper reflective retinal spots and visual function after anti-VEGF treatment in DME patients, it was reported that hyper reflective retinal spots decreased after anti-VEGF treatment and this was found to be correlated with increased retinal sensitivity (17). In our study, the patients were divided into 
2 groups in regard to their visual outcomes and several OCT parameters were compared between the two groups. The presence of an intact IS/OS band and subfoveal neuro sensory retinal detachment were both arithmetically more frequent in group 1, none of the OCT parameters did not show statistically significance. An intact IS/OS band was presence in $76.5 \%$ of the patients in group 1, and in $42.9 \%$ of the patients in group. Interestingly subfoveal sensorial retinal detachment was present in $52.9 \%$ of the patients in group 1, and in only $14.4 \%$ of the patients in group 2 . Although the presence of macular ischemia was not a main outcome measure of the study, we evaluated it between the two groups and it was found statistically more frequent in group 2.

The main limitation of the study was the low patient number. The duration of diabetes and DME were not assessed. One other limitation was that we did not classify the patients according to the severity of non-proliferative DR. However, all patients were treatment naïve and we evaluated the patients with only low baseline visual acuity which were the strengths of the study. Also the study consisted of the real life data regarding about the patient compliance to visits and injections.

\section{References}

1. Jusufbegovic D, M ugavin M O, Schaal S. Evolution of controlling diabetic retinopathy: changing trends in the management of diabetic macular edema at a single institution over the past decade. Retina 2015; 35:929-34.

2. Early Treatment Diabetic Retinopathy Study Research Group: Photocoagulation for diabetic macular edema: ETDRS report No.4. Int Ophtahlmol Clin 1987; 27:265-72.

3. Ozkaya A, AlagozC, Alagoz N, Gunes H, Yilmazl, Perente I, et al. Dexamethasone implant in pseudophakic and nonglaucomatous subgroup of diabetic macular edema patients: a real life experience. Eur J Ophthalmol. 2016;26:351-5.

4. Fechter $\mathrm{C}$, Frazier $\mathrm{H}$, Marcus WB, Faroog A, Singh $\mathrm{H}$, Marcus DM. Ranibizumab $0.3 \mathrm{mg}$ for persistent diabetic macular edema after recent, frequent, and chronic bevacizumab: The Rotate Trial. Ophthalmic Surg Lasers Imaging Retina 2016;47:1-18.

5. Elman MJ, Aiello LP, Beck RW, Bressler NM, Bressler SB, Edwards AR, et al. Diabetic Retinopathy Clinical Research Network. randomized trial evaluating ranibizumab plus prompt or deferred laser or triamcinolone plus prompt laser for diabetic macular edema. Ophthalmology 2010;117:1064-1077.

6. Nguyen QD, Brown DM , M arcus DM, Boyer DS, Patel S, Feiner

\section{Conclusion}

In conclusion ranibizumab seems to be effective in the treatment of DME patients with a low visual acuity. Although as needed treatment regimen seems to be insufficient in real life, both visual and anatomical outcomes were acceptable. However, under-treatment of these patients probably limited the visual success in this group of patients. None of the evaluated OCT parameters seemed to affect the visual outcomes of ranibizumab treatment. Only the presence of macular ischemia at the baseline might have a negative effect on visual outcomes. As a result this manuscript revealed some useful data which might be useful in the treatment of DME patients with low visual acuity which are; ranibizumab treatment may be beneficial in most of the patients in this subgroup, none of the evaluated OCT parameters do not seem to be associated with the visual outcomes, but macular ischemia seems to be associated with worse visual outcome.

\section{Acknowledgements}

The authors thank to Dr. Hande M efkure Ozkaya for her to review the manuscript for English editing.

L, et al. Ranibizumab for diabetic macular edema: results from 2 phase III randomized trials: RISE and RIDE. Ophthalmology 2012;119:789-801.

7. Payne JF, Wykoff CC, Clark WL, Bruce BB, Boyer DS, Brown DM, et al; TREX-DM E Study Group. Randomized Trial of Treat and Extend Ranibizumab with and without Navigated Laser for Diabetic Macular Edema: TREX-DME 1 Year Outcomes. Ophthalmology. 2017;124:74-81.

8. M enchini U, Bandello F, De Angelis V, Ricci F, Bonavia L, Viola F, et al. Ranibizumab for visual impairment due to diabetic macular edema: realworld evidence in the Italian population (PRIDEStudy).J Ophthalmol 2015;2015:324841.

9. Vorum H, Kruse Olesen T, Zinck J, Størling Hedegaard M. Real world evidence of use of anti-VEGF therapy in Denmark. Current Medical Research and Opinion 2016;32:1943-1950.

10.M urakami T, Yoshimura N. Structural changes in indiviual retinal layers in diabetic macular edema. J Diabetes Res 2013;2013:920713.

11.Sophie R, Lu N, Campochiaro PA. Predictors of functional and anatomic outcomes in patients with diabetic macular edema treated with ranibizumab. Ophthalmology 2015;122:13951401.

12.Radwan SH, Soliman AZ, Tokarev J, Zhang L, van Kujik FJ, Koozekanani DD. Association of disorganization of retinal inner 
layers with vision after resolution of center-involved diabetic macular edema. JAM A Ophthalmol 2015;133:820-825.

13.Seo KH, Yu SY, Kim M, Kwak HW. Visual and morphological outcomes of intravitreal ranibizumab for diabetic macular edema based on optical coherence tomography patterns. Retina 2016;36:588-595.

14.Ozkaya A, Alkin Z, Agca A, Satici T, Karakucuk Y, Yazici AT, et al. One-year results of treatment with bevacizumab alone or ranibizumab alone for low visual acuity due to neovascular age-related macular degeneration. J Ocul Pharmacol Ther 2013;29:865-869.

15.Ruia S, Saxena S, Gemmy Cheung CM, Gilhotra JS, Lai TY. Spectral domain optical coherence tomography features and classification systems for diabetic macular edema: A review.
Asia Pac J Ophthalmol (Phila) 2016;5:360-367.

16.Ozkaya A, Alkin Z, Karakucuk Y, Karatas G, Fazil K, Gurkan Erdogan $M$, et al. Thickness of the retinal photoreceptor outer segment layer in healthy volunteers and in patients with diabetes mellitus without retinopathy, diabetic retinopathy, or diabetic macular edema. SaudiJ Ophthalmol 2017;31:69-75.

17.Vujosevic S, Berton M, Bini S, Casciano M, Cavarzeran F, $M$ idena $E$. Hyperreflective retinal spots and visual function after anti-vascular endothelial growth factor treatment in center-involving diabetic macular edema. Retina 2016;36:1298-1308.

18. Bresnick GH, Condit R, Syrjala S, Palta M, Groo A, Korth K. Abnormalities of the foveal avascular zone in diabetic retinopathy. Arch Ophthalmol 1984;102:1286-93. 